J. Nonlinear Var. Anal. 4 (2020), No. 2, pp. 207-223

Available online at http://jnva.biemdas.com

https://doi.org/10.23952/jnva.4.2020.2.04

\title{
PRIMAL-DUAL PARTITIONS IN LINEAR SEMI-INFINITE PROGRAMMING WITH BOUNDED COEFFICIENTS
}

\author{
ABRAHAM B. BARRAGÁN ${ }^{1}$, LIDIA A. HERNÁNDEZ ${ }^{2}$, ALFREDO N. IUSEM ${ }^{3, *}$, MAXIM I. TODOROV ${ }^{4, \dagger}$ \\ ${ }^{1}$ Facultad de Ciencias Físico Matemáticas, UANL, Monterrey, Nuevo León, Mexico \\ ${ }^{2}$ Facultad de Ciencias Físico Matemáticas, BUAP, Puebla, Mexico \\ ${ }^{3}$ Instituto de Matemática Pura e Aplicada, IMPA, Rio de Janeiro, Brazil \\ ${ }^{4}$ Department of Actuarial Sciences, Physics and Mathematics, \\ UDLAP, 72820 San Andrés Cholula, Puebla, Mexico
}

\begin{abstract}
We consider two partitions over the space of linear semi-infinite programming parameters with a fixed index set and bounded coefficients (the constraint functions are bounded). The first one is the primal-dual partition inspired by consistency and boundedness of the optimal value of the problem. The second one is a refinement of the primal-dual partition that arises by considering also the boundedness of the optimal set. These two partitions have been studied in the continuous case, i.e., when the set of indices is an infinite compact topological space and the constraint functions are continuous. In this paper, we extend these results to the case in which the constraint functions are bounded, but not necessarily continuous. We study the same primal-dual partitions and characterize the interior of the corresponding cells. Through examples, we show that the conditions characterizing the cells of both partitions in the continuous case are neither necessary nor sufficient when the constraint functions are just bounded. In addition, a sufficient condition for the boundedness of the optimal set of the dual problem is established.
\end{abstract}

Keywords. Linear semi-infinite programming; Bounded linear semi-infinite optimization problems; Primal-dual partition.

\section{INTRODUCTION}

Let $T$ be an arbitrary set. Define $B(T)$ and $B(T)^{n}$ as the sets of bounded functions $b: T \rightarrow \mathbb{R}$ and $\boldsymbol{a}: T \rightarrow \mathbb{R}^{n}$, respectively, meaning that there exists $\tau \in \mathbb{R}$ such that $\|\boldsymbol{a}(t)\| \leq \tau$ and $|b(t)| \leq \tau$ for all $t \in T$.

We will also consider the particular case in which $T$ is a compact topological space, in which case $C(T)$, and $C(T)^{n}$ denote the sets of continuous functions defined on $T$ with values in $\mathbb{R}$ and $\mathbb{R}^{n}$ respectively. We will refer to this case as the continuous case.

Define $\Pi=B(T)^{n} \times B(T) \times \mathbb{R}$. Note that $\Pi$ becomes a normed space with the norm $\|\cdot\|$ defined as

$$
\|\pi\|:=\max \left\{\|\boldsymbol{c}\|_{\infty}, \sup _{t \in T}\left\|\left(\begin{array}{l}
\boldsymbol{a}_{t} \\
b_{t}
\end{array}\right)\right\|_{\infty}\right\},
$$

\footnotetext{
${ }^{*}$ Corresponding author. ${ }^{\dagger}$ On leave from IMI-BAS, Sofia, Bulgaria.

E-mail addresses: ab_beni211@ @otmail.com (A.B. Barragán), lhernan@fcfm.buap.mx (L.A. Hernández), iusp @impa.br (A.N. Iusem), maxim.todorov@udlap.mx (M.I. Todorov).

Received May 29, 2019; Accepted February 12, 2020.
}

(C)2020 Journal of Nonlinear and Variational Analysis 
where $\|\cdot\|_{\infty}$ indicates the supremum norm. Obviously, the same holds for $C(T)^{n} \times C(T) \times \mathbb{R}$ in the continuous case by the compactness of $T$.

We associate with each triplet $\pi \in \Pi$ a primal problem

$$
\begin{array}{ll}
P: & \quad \inf \boldsymbol{c}^{\prime} \boldsymbol{x} \\
& \text { s.t. } \boldsymbol{a}_{t}^{\prime} \boldsymbol{x} \geq b_{t}, t \in T,
\end{array}
$$

and a dual problem, in the sense of Haar,

$$
\begin{gathered}
D: \quad \sup \sum_{t \in T} \lambda_{t} \boldsymbol{b}_{t} \\
\text { s.t. } \sum_{t \in T} \lambda_{t} \boldsymbol{a}_{t}=\boldsymbol{c} \\
\lambda \in \mathbb{R}_{+}^{(T)} .
\end{gathered}
$$

Above and henceforth, $\mathbb{R}_{+}^{(T)}$ denotes the set of nonnegative general finite sequences, meaning the functions $\lambda: T \rightarrow \mathbb{R}_{+}$satisfying that $\lambda_{t}=0$ for all $t \in T$ except possibly for a finite number of indices. In $\mathbb{R}_{+}^{(T)}$, we consider the norms $\ell_{\infty}$ and $\ell_{1}$.

As both the primal and the dual problem are defined with the same data $a, b$ and $c$, they are represented by the triplet $\pi:=(\boldsymbol{a}, b, \boldsymbol{c})$.

We partition $\Pi$ in several sets depending on the properties of the primal and dual problem defined by an element $\pi \in \Pi$, i.e., whether the problem is or not solvable, its optimal value is or not bounded, and the set of solutions is or not bounded.

The partitions where $T$ is a compact topological space and the functions $\boldsymbol{a}$ and $b$ are continuous (i.e., the continuous case), have been analyzed in $[1,5,6]$. In particular, the latter reference deals with the consistence and boundedness of the optimal value of the problems and these properties define the primal-dual partition. The interior of the sets generated through the partition was also studied in these references. In [8], partitions corresponding to an arbitrary index set $T$ (not necessarily a topological space), and arbitrary functions $\boldsymbol{a}$ and $b$ were analyzed.

Throughout this paper, we assume that the functions $\boldsymbol{a}$ and $b$ are bounded.

In the present paper, a refinement of the primal-dual partition is presented. The new partition considers also the boundedness of the set of solutions of the problem. Hence, this work extends the study of the primal-dual partition and its refinement to the case of bounded (rather than continuous) coefficients.

The new results are presented in Sections 3 and 4. In Section 3, we characterize the interior of the sets that are generated by the primal-dual partition and show that the characterization is similar to the one which holds in the continuous case. In Section 4, we show that the conditions that characterize the sets generated by the refinement in the continuous case are neither necessary nor sufficient in the bounded case. In addition, we present a condition that implies the boundedness of the optimal set of the dual problem in the bounded case.

\section{PRELIMINARIES}

We begin with the notation used in the paper. We denote by $\mathbb{R}_{+}$the set of positive real numbers. In the $n$-dimensional space $\mathbb{R}^{n}$ endowed with the Euclidean norm, $\boldsymbol{x}$ ' stands for the transpose of the vector column $\boldsymbol{x}$ and the null vector will be denoted by $\boldsymbol{0}_{n}$. If $X$ is a set of any topological space, then int $X$ and $c l X$ denote its interior and its closure, respectively. Given a 
nonempty set $X \subset \mathbb{R}^{n}$, conv $X$ and cone $X$ denote its convex and conical hull, respectively. If $C$ is a nonempty convex set, then its recession cone $O^{+}(C)$ is defined as:

$$
\text { cone }\left\{\boldsymbol{y} \in \mathbb{R}^{n}: \boldsymbol{x}+\alpha \boldsymbol{y} \in C \text { for all } \boldsymbol{x} \in C \text { and for all } \alpha>0\right\} \text {. }
$$

The feasible (optimal) set of $P$ and $D$ are denoted as $F\left(F^{*}\right)$ and $\Lambda\left(\Lambda^{*}\right)$, respectively. The optimal value of the primal (dual) problem $P(D)$ are denoted as $v^{P}(\pi)\left(v^{D}(\pi)\right)$ where, as usual, $v^{P}(\pi)=+\infty$ and $v^{D}(\pi)=-\infty$ when the corresponding problems become inconsistent.

With each $\pi$ we associate the first and second moment cones

$$
\begin{gathered}
M:=\text { cone }\left\{\boldsymbol{a}_{t}, t \in T\right\}, \\
N:=\text { cone }\left\{\left(\boldsymbol{a}_{t}, b_{t}\right)^{\prime}, t \in T\right\},
\end{gathered}
$$

and its characteristic cone

$$
K:=\text { cone }\left\{\left(\boldsymbol{a}_{t}, b_{t}\right)^{\prime}, t \in T ;\left(\boldsymbol{0}_{n},-1\right)^{\prime}\right\} .
$$

Recall that $\pi$ satisfies the Slater condition if there exists $\overline{\boldsymbol{x}} \in \mathbb{R}^{n}$ such that $\boldsymbol{a}_{t}^{\prime} \overline{\boldsymbol{x}}>b_{t}$ for all $t \in T$. Also, $\pi$ satisfies the strong Slater condition if there exist $\varepsilon>0$ and $\overline{\boldsymbol{x}} \in \mathbb{R}^{n}$, such that $\boldsymbol{a}_{t}^{\prime} \overline{\boldsymbol{x}} \geq b_{t}+\varepsilon$ for all $t \in T$. From these definitions, we get that every parameter that satisfies the strong Slater condition is consistent and satisfies the Slater condition. However, the converse statement is not true in general (see Example 4.5). In [4, Theorem 6.1], it was shown that a parameter satisfies the strong Slater condition if and only if

$$
\boldsymbol{0}_{n+1}:=\left(\boldsymbol{0}_{n}, 0\right)^{\prime} \notin c l G,
$$

where

$$
G:=\operatorname{conv}\left\{\left(\boldsymbol{a}_{t}, b_{t}\right)^{\prime}, t \in T\right\} .
$$

It is worth mentioning that in the continuous case the Slater and the strong Slater conditions coincide.

We will denote by $\Pi_{C}^{P}, \Pi_{I C}^{P}, \Pi_{B}^{P}$ and $\Pi_{U B}^{P}\left(\Pi_{C}^{D}, \Pi_{I C}^{D}, \Pi_{B}^{D}\right.$ and $\left.\Pi_{U B}^{D}\right)$ the sets of parameters that have primal (dual) problem consistent, inconsistent, bounded (consistent with finite optimal value) and unbounded, respectively. Also, $\Pi_{S}^{P}\left(\Pi_{S}^{D}\right)$ will denote the set of parameters with the solvable primal (dual) problem which has the bounded optimal set, while $\Pi_{N}^{P}\left(\Pi_{N}^{D}\right)$ will denote the set of parameters with the primal (dual) problem, which is not solvable or has unbounded optimal set.

In the continuous case, the sets $\Pi_{S}^{P}$ and $\Pi_{S}^{D}$ were characterized in [5]. These characterizations are presented in the next lemma.

\section{Lemma 2.1.}

(i) $\pi \in \Pi_{S}^{P}$ if and only if $\left(\begin{array}{c}\boldsymbol{0}_{n} \\ 1\end{array}\right) \notin \mathrm{cl} K$ and $\boldsymbol{c} \in$ int $M$;

(ii) $\pi \in \Pi_{S}^{D}$ if and only if $c \in M$ and $\pi$ satisfies the Slater condition.

In the first primal-dual partition, presented in [6], the primal and dual problems are classified in inconsistent (IC), bounded (B) and unbounded (UB) classes. This partition is showed in the following table. 


\begin{tabular}{|c|c|c|c|}
\hline$(D) \backslash(P)$ & $I C$ & $B$ & $U B$ \\
\hline$I C$ & $\Pi_{4}$ & $\Pi_{5}$ & $\Pi_{2}$ \\
\hline$B$ & $\Pi_{6}$ & $\Pi_{1}$ & \\
\hline$U B$ & $\Pi_{3}$ & & \\
\hline
\end{tabular}

Table 1

where,

$$
\begin{gathered}
\Pi_{1}:=\Pi_{B}^{P} \cap \Pi_{B}^{D}, \Pi_{2}:=\Pi_{U B}^{P} \cap \Pi_{I C}^{D}, \quad \Pi_{3}:=\Pi_{I C}^{P} \cap \Pi_{U B}^{D}, \\
\Pi_{4}:=\Pi_{I C}^{P} \cap \Pi_{I C}^{D} \Pi_{5}:=\Pi_{B}^{P} \cap \Pi_{I C}^{D}, \text { and } \Pi_{6}:=\Pi_{I C}^{P} \cap \Pi_{B}^{D} .
\end{gathered}
$$

We conclude this section with the characterization of the sets $\Pi_{i}, i=1, \ldots, 6$. Here, $M, N$ and $K$ play a crucial role (see [6]). The next theorem, proved in [8], holds for the general linear semi-infinite optimization, hence in the particular case when $\boldsymbol{a}$ and $b$ are bounded.

Theorem 2.1. $\quad$ i) $\pi \in \Pi_{1}$ if and only if $\left(\boldsymbol{O}_{n}, 1\right)^{\prime} \notin \mathrm{cl} N$ and $\boldsymbol{c} \in M$.

ii) $\pi \in \Pi_{2}$ if and only if $\left(\boldsymbol{0}_{n}, 1\right)^{\prime} \notin \operatorname{cl} N$ and $(\{\boldsymbol{c}\} \times \mathbb{R}) \cap \operatorname{cl} N=\emptyset$.

iii) $\pi \in \Pi_{3}$ if and only if $\{\boldsymbol{c}\} \times \mathbb{R} \subseteq K$.

iv) $\pi \in \Pi_{4}$ if and only if $\left(\boldsymbol{0}_{n}, 1\right)^{\prime} \in \operatorname{cl} N$ and $\boldsymbol{c} \notin M$.

v) $\pi \in \Pi_{5}$ if and only if $\boldsymbol{c} \notin M,\left(\boldsymbol{0}_{n}, 1\right)^{\prime} \notin \mathrm{cl} N$ and $(\{\boldsymbol{c}\} \times \mathbb{R}) \cap \mathrm{cl} N \neq \emptyset$.

vi) $\pi \in \Pi_{6}$ if and only if $\left(\boldsymbol{0}_{n}, 1\right)^{\prime} \in \operatorname{cl} N, \boldsymbol{c} \in M$ and $\{\boldsymbol{c}\} \times \mathbb{R} \nsubseteq K$.

\section{Primal-DUAL Stability}

The following theorem, established in [7], presents the characterization of the interior of the sets generated by the primal-dual partition in the continuous case, i.e., when the constraint functions are continuous.

Theorem 3.1. Consider the continuous case and let $\pi \in C(T)^{n} \times C(T) \times \mathbb{R}$ be a parameter. The following assertions hold:

i) $\pi \in$ int $\Pi_{1}$ if and only if $\pi$ satisfies the Slater condition and $c \in$ int $M$.

ii ) $\pi \in$ int $\Pi_{2}$ if and only if there exists $\boldsymbol{y} \in \mathbb{R}^{n}$ such that $\boldsymbol{c}^{\prime} \boldsymbol{y}<0$ and $\boldsymbol{a}_{t}^{\prime} \boldsymbol{y}>0$, for all $t \in T$.

iii) $\pi \in$ int $\Pi_{3}$ if and only if $\left(\boldsymbol{0}_{n}, 1\right)^{\prime} \in$ int $N$.

iv) int $\Pi_{i}=\emptyset$ for $i=4,5,6$.

The following theorem, obtained in [9], characterizes the interior of the sets generated by the primal-dual partition in the general case, i.e. when $T$ is an arbitrary set and no assumptions are made on the constraint functions (not even boundedness).

Theorem 3.2. Take $\pi \in \Pi$. The following assertions hold:

i) $\pi \in$ int $\Pi_{1}$ if and only if $\pi$ satisfies the strong Slater condition and $c \in$ int $M$. 
ii) $\pi \in$ int $\Pi_{2}$ if and only if there exist $\boldsymbol{y} \in \mathbb{R}^{n}$ and $\delta>0$ such that $\boldsymbol{c}^{\prime} \boldsymbol{y}<0$ and $\boldsymbol{a}_{t}^{\prime} \boldsymbol{y} \geq$ $\delta$, for all $t \in T$.

iii) (a) $\pi \in$ int $\Pi_{3}, M=\mathbb{R}^{n}$ if and only if $\left(\boldsymbol{0}_{n}, 1\right)^{\prime} \in$ int $N$.

(b) $\pi \in$ int $\Pi_{3}, M \neq \mathbb{R}^{n}$ if and only if $\boldsymbol{0}_{n} \notin$ int $\operatorname{conv}\left\{\boldsymbol{a}_{t}: t \in T\right\},\left(\boldsymbol{0}_{n}, 1\right)^{\prime} \in O^{+}(c l G), \boldsymbol{c} \in$ int $M$.

(c) $\pi \in$ int $\Pi_{4}$ if and only if $\boldsymbol{0}_{n} \notin c l \operatorname{conv}\left\{\boldsymbol{a}_{t}: t \in T\right\},\left(\boldsymbol{0}_{n}, 1\right)^{\prime} \in O^{+}(c l G)$ and $\boldsymbol{c} \in$ int $M$. iv) int $\Pi_{i}=\emptyset$ for $i=5,6$.

In the remainder of this section, we present new results concerning the primal-dual partition in the bounded case.

The following theorem shows that the characterization of the interior of the sets generated by the primal-dual partition, in the case of bounded coefficients, is similar to the corresponding results for the continuous case. However, in the bounded case the Slater condition is replaced by the strong Slater condition, because in the case of bounded coefficient there are parameters that satisfy the Slater condition, but not the strong Slater condition (see Example 4.5).

Theorem 3.3. Let $\pi=(\boldsymbol{a}, b, \boldsymbol{c})$ be a parameter with bounded coefficients. Then

i) $\pi \in$ int $\Pi_{1}$ if and only if $\pi$ satisfies the strong Slater condition and $\boldsymbol{c} \in$ int $M$.

ii) $\pi \in$ int $\Pi_{2}$ if and only if there exists $\boldsymbol{y} \in \mathbb{R}^{n}$ such that, $\boldsymbol{c}^{\prime} \boldsymbol{y}<0$ and $\boldsymbol{a}_{t}^{\prime} \boldsymbol{y}>0$ for all $t \in T$.

iii) $\pi \in$ int $\Pi_{3}$ if and only if $\left(\boldsymbol{0}_{n}, 1\right)^{\prime} \in$ int $N$.

iv) int $\Pi_{i}=\emptyset$ for $i=4,5,6$.

The proof of Theorem 3.3 follows from Theorems 3.1, 3.2, and the next observation.

Observation 3.4. If $\boldsymbol{a}$ and $b$ are bounded, then $O^{+}(c l G)=\left\{\boldsymbol{0}_{n+1}\right\}$.

Note that in the case of bounded coefficients, $\pi \in$ int $\Pi_{3}$ and $M \neq \mathbb{R}^{n}$, which is impossible because otherwise $\left(\boldsymbol{0}_{n}, 1\right)^{\prime} \in O^{+}(\mathrm{cl} G)$ (see Theorem 3.2), and in this case, $O^{+}(c l G)=\left\{\boldsymbol{0}_{n+1}\right\}$.

In the same way, we get that int $\Pi_{4}=\emptyset$.

\section{FIRST REFINED PRIMAL-DUAL PARTITION}

A refinement of the primal-dual partition follows from classifying the bounded primal and dual problems in two categories. The first one is formed by solvable problems with bounded optimal set $(S)$. The second one includes unsolvable problems and those that have unbounded optimal set $(N)$. The refinement is called refined primal-dual partition and it is shown in Table 2. 


\begin{tabular}{|c|c|c|c|c|}
\hline \multirow[t]{2}{*}{$D \backslash P$} & \multirow[t]{2}{*}{ IC } & \multicolumn{2}{|c|}{ B } & UB \\
\hline & & $S$ & $\mathrm{~N}$ & \\
\hline IC & $\Pi_{4}$ & $\Pi_{5}^{1}$ & $\Pi_{5}^{2}$ & $\Pi_{2}$ \\
\hline S & $\Pi_{6}^{1}$ & $\Pi_{1}^{1}$ & $\Pi_{1}^{3}$ & \\
\hline $\mathrm{N}$ & $\Pi_{6}^{2}$ & $\Pi_{1}^{2}$ & $\Pi_{1}^{4}$ & \\
\hline UB & $\Pi_{3}$ & & & \\
\hline
\end{tabular}

Table 2

In the refinement,

$$
\begin{aligned}
& \Pi_{1}^{1}:=\Pi_{S}^{P} \cap \Pi_{S}^{D}, \Pi_{1}^{2}:=\Pi_{S}^{P} \cap \Pi_{N}^{D}, \\
& \Pi_{1}^{3}:=\Pi_{N}^{P} \cap \Pi_{S}^{D}, \Pi_{1}^{4}:=\Pi_{N}^{P} \cap \Pi_{N}^{D}, \\
& \Pi_{5}^{1}:=\Pi_{S}^{P} \cap \Pi_{I C}^{D}, \Pi_{5}^{2}:=\Pi_{N}^{P} \cap \Pi_{I C}^{D}, \\
& \Pi_{6}^{1}:=\Pi_{I C}^{P} \cap \Pi_{S}^{D} \text { and } \Pi_{6}^{2}:=\Pi_{I C}^{P} \cap \Pi_{N}^{D} .
\end{aligned}
$$

The other sets are the same as in the primal-dual partition.

According to the Duality Theorem [3, Theorem 4.2], in ordinary linear programming, we have $\Pi_{1}^{i}=\emptyset$ for $i=2,3,4$. However, in the case of bounded coefficients, these sets may be nonempty, as shown in Theorem 3.1 of [6].

The conditions characterizing the sets generated by the refined primal-dual partition in the continuous case, are as follows:

Theorem 4.1. [5, Theorem 3.3] The following statements are true:

i) $\pi \in \Pi_{1}^{1}$ if and only if $c \in$ int $M$ and $\pi$ satisfies the Slater condition;

ii) $\pi \in \Pi_{1}^{2}$ if and only if $\left(\begin{array}{c}\boldsymbol{0}_{n} \\ 1\end{array}\right) \notin \mathrm{cl} K, \boldsymbol{c} \in$ int $M$ and $\pi$ does not satisfy the Slater condition;

iii) $\pi \in \Pi_{1}^{3}$ if and only if $c \in M \backslash$ int $M$ and $\pi$ satisfies the Slater condition;

iv) $\pi \in \Pi_{1}^{4}$ if and only if $\left(\begin{array}{c}\boldsymbol{0}_{n} \\ 1\end{array}\right) \notin c l K, c \in M \backslash$ int $M$ and $\pi$ does not satisfy the Slater condition.

The condition that characterizes the set $\Pi_{S}^{P}$, presented in Lemma 2.1, holds true in the case of bounded coefficients. For the dual problem, this is not the case: the condition characterizing $\Pi_{S}^{D}$ fails for bounded coefficients, as shown in the next example, where $c^{1} \in M_{1}$ and $\pi^{1}$ satisfies the Slater condition, but the dual problem associated with the parameter $\pi^{1}$ is unsolvable. 
Example 4.1. Let $T=[0,1]$ and $n=2$. We define $\pi^{1}:=\left(\boldsymbol{a}^{1}, b^{1}, \boldsymbol{c}^{1}\right)$ such that $\boldsymbol{a}_{t}^{1}:=(t, 1)$ for all $t \in T$,

$$
b_{t}^{1}:= \begin{cases}1, & \text { if } t=0 \\ 0, & \text { if } 0<t<1 \\ -1, & \text { if } t=1,\end{cases}
$$

and $c^{1}:=(1 / 3,1)$. In [7], it was shown that $c^{1} \in M_{1}$ and $\pi^{1}$ satisfies the Slater condition, but the dual problem is not solvable.

In Example 4.1, the dual problem is unsolvable, so that the optimal set of the dual problem is empty, and henceforth bounded. In addition, the parameter satisfies the strong Slater condition. In fact, for $\varepsilon=\frac{1}{2}$, the point $\left(\begin{array}{l}0 \\ 2\end{array}\right)$ is a strong Slater point, which suggests the following result: if $c$ belongs to $M$ and $\pi$ satisfies the strong Slater condition, then $\Lambda^{*}$ is bounded. The proof of this statement requires a result analogous to Carathéodory's Theorem (see, [9, Corollary 17.1.2]), but for positive, rather than arbitrary, linear combinations.

Lemma 4.1. If $\sum_{t \in T} \lambda_{t} \boldsymbol{a}_{t}=\boldsymbol{c}$ with $\lambda_{t} \geq 0$ for all $t \in T$, then there exist $\gamma_{1}, \ldots, \gamma_{n+1} \in \mathbb{R}$ and, $t_{1}, \ldots, t_{n+1} \in T$ such that $\sum_{i=1}^{n+1} \gamma_{i} \boldsymbol{a}_{t_{i}}=\boldsymbol{c}$ and $\sum_{t \in T} \lambda_{t}=\sum_{i=1}^{n+1} \gamma_{i}$.

Proof. Let $\sum_{t \in T} \lambda_{t} \boldsymbol{a}_{t}=\boldsymbol{c}$. The case $\sum_{t \in T} \lambda_{t}=0$ is obvious. Now, if $\sum_{t \in T} \lambda_{t}=\lambda>0$, then

$$
\sum_{t \in T} \frac{\lambda_{t}}{\lambda} a_{t}=\frac{c}{\lambda}
$$

and

$$
\sum_{t \in T} \frac{\lambda_{t}}{\lambda}=1
$$

From Carathéodory Theorem for convex combinations ([9, Theorem 17.1]), there exist $\beta_{1}, \ldots, \beta_{n+1} \in$ $\mathbb{R}_{+}$such that

$$
\sum_{i=1}^{n+1} \beta_{i} a_{t_{i}}=\frac{c}{\lambda},
$$

with $\sum_{i=1}^{n+1} \beta_{i}=1$. Multiplying the above equalities by $\lambda$ yields

$$
\sum_{i=1}^{n+1} \lambda \beta_{i} a_{t_{i}}=c
$$

with $\sum_{i=1}^{n+1} \lambda \beta_{i}=\lambda$. Taking $\gamma_{i}=\lambda \beta_{i}$, we conclude that $\sum_{i=1}^{n+1} \gamma_{i} \boldsymbol{a}_{t_{i}}=\boldsymbol{c}$ and $\sum_{i=1}^{n+1} \gamma_{i}=\sum_{t \in T} \lambda_{t}$.

Next we prove the above announced result.

Theorem 4.2. Let $\pi$ a parameter with $|T| \geq n+2$. If $c \in M$ and $\pi$ satisfies the strong Slater condition, then $\Lambda^{*}$ is bounded in the norm $\ell_{1}$.

Proof. Since $c \in M$, we get that $\pi \in \Pi_{C}^{D}$. Also, since $\pi$ satisfies the strong Slater condition, we have $\pi \in \Pi_{C}^{P}$. It follows that $\pi \in \Pi_{1}$, and in particular, $\pi \in \Pi_{B}^{D}$. 
Now, if $\Lambda^{*}=\emptyset$, the result is obvious. Assume that $\Lambda^{*} \neq \emptyset$ and $\Lambda^{*}$ is not bounded. Take $\left\{\lambda^{m}\right\}$ in $\Lambda^{*}$ such that $\sum_{t \in T} \lambda_{t}^{m} \rightarrow \infty$. Since $\Lambda^{*} \subseteq \Lambda$ and $\lambda^{m}$ belongs to $\Lambda^{*}$ for all $m$, we have

$$
\sum_{t \in T} \lambda_{t}^{m}\left(\begin{array}{l}
\boldsymbol{a}_{t} \\
b_{t}
\end{array}\right)=\left(\begin{array}{c}
\boldsymbol{c} \\
v^{D}(\pi)
\end{array}\right)
$$

From (4.1), we get that, for each $\left.m, \underset{{ }_{v} D(\pi)}{\boldsymbol{c}}\right)$ is a positive linear combination of $\left\{\left(\boldsymbol{a}_{t}, b_{t}\right)^{\prime}, t \in T\right\}$. By Lemma 4.1, we obtain that for all $m$ there exist $\beta_{1}^{m}, \ldots, \beta_{n+2}^{m} \in \mathbb{R}_{+}$such that

$$
\sum_{i=1}^{n+2} \beta_{i}^{m}\left(\begin{array}{c}
\boldsymbol{a}_{t_{i}}^{m} \\
b_{t_{i}}^{m}
\end{array}\right)=\left(\begin{array}{c}
\boldsymbol{c} \\
v^{D}(\pi)
\end{array}\right)
$$

with

$$
\sum_{i=1}^{n+2} \beta_{i}^{m}=\sum_{t \in T} \lambda_{t}^{m}
$$

In view of (4.2),

$$
\sum_{i=1}^{n+2} \frac{\beta_{i}^{m}}{\sum_{i=1}^{n+2} \beta_{i}^{m}}\left(\begin{array}{c}
\boldsymbol{a}_{t_{i}}^{m} \\
b_{t_{i}}^{m}
\end{array}\right)=\frac{1}{\sum_{i=1}^{n+2} \beta_{i}^{m}}\left(\begin{array}{c}
\boldsymbol{c} \\
v^{D}(\pi)
\end{array}\right)
$$

Since $\left\{\frac{\beta_{i}^{m}}{\sum_{i=1}^{n+2} \beta_{i}^{m}}\right\}$ and $\left\{\left(\boldsymbol{a}_{t_{i}}^{m}, b_{t_{i}}^{{ }^{\prime}}\right)\right\}$ are bounded, if $m \rightarrow \infty$ in (4.4), it follows from (4.4) and (4.3) that $\sum_{i=1}^{n+2} \beta_{i}\left(\begin{array}{c}a_{i} \\ b_{i}\end{array}\right)=\left(\begin{array}{c}\boldsymbol{o}_{n} \\ 0\end{array}\right)$, with $\sum_{i=1}^{m+2} \beta_{i}=1$, which implies that

$$
\left(\begin{array}{c}
\boldsymbol{O}_{n} \\
0
\end{array}\right) \in \operatorname{cl} \operatorname{conv}\left\{\left(\begin{array}{l}
\boldsymbol{a}_{t} \\
b_{t}
\end{array}\right): t \in T\right\} .
$$

It follows that the strong Slater condition fails, in contradiction with the hypothesis.

Corollary 4.1. If $\Lambda^{*}$ is not bounded in the norm $\ell_{1}$ and $|T| \geq n+2$, then $\pi$ does not satisfy the strong Slater condition.

We recall that if $\Lambda^{*}$ is bounded in the norm $\ell_{1}$, then it is also bounded in the norm $\ell_{\infty}$. We also mention that the condition $|T| \geq n+2$ is rather harmless, since otherwise $T$ is finite and we are in the classical linear programming setting.

Theorem 4.2 implies, that under certain conditions, sup $\left\{\|\lambda\|_{1}, \lambda \in \Lambda^{*}\right\}<\infty$. In the following theorem, we show that if $\boldsymbol{c}=\boldsymbol{0}_{n}$, then $\sup \left\{\|\lambda\|_{1}, \lambda \in \Lambda^{*}\right\}=0$.

Theorem 4.3. Take $\pi=(\boldsymbol{a}, b, \boldsymbol{c})$ such that $\boldsymbol{c}=\boldsymbol{0}_{n}$ and $|T| \geq n+2$. If $\boldsymbol{c} \in$ int $M$ and $\pi$ satisfies the strong Slater condition, then $\Lambda^{*}=\{\lambda \equiv 0\}$.

Proof. First, since $c \in M$ and $\pi$ satisfies the strong Slater condition, Theorem 4.2 implies that $\Lambda^{*}$ is bounded. On the other hand, since the primal problem is consistent and $c \in$ int $M$, we have that $v^{P}(\pi)=v^{D}(\pi)$ in view of [4, Theorem 8.1]. Moreover, since $\boldsymbol{c}=\boldsymbol{0}_{n}, v^{D}(\pi)=0$.

Now, suppose that $\Lambda^{*}=\emptyset$. In this case, the function $\lambda \equiv 0$ is an optimal solution, which contradicts the assumption. 
Since $\Lambda^{*} \neq \emptyset$, we take $\lambda \in \Lambda^{*}$ and suppose that $\sum_{t \in T} \lambda_{t}>0$. Because $\boldsymbol{c}=\boldsymbol{0}_{n}$, we have

$$
\sum_{t \in T} \lambda_{t}\left(\begin{array}{c}
\boldsymbol{a}_{t} \\
b_{t}
\end{array}\right)=\left(\begin{array}{c}
\boldsymbol{0}_{n} \\
0
\end{array}\right)
$$

Then,

$$
\sum_{t \in T} \frac{\lambda_{t}}{\sum_{t \in T} \lambda_{t}}\left(\begin{array}{c}
\boldsymbol{a}_{t} \\
b_{t}
\end{array}\right)=\left(\begin{array}{c}
\boldsymbol{0}_{n} \\
0
\end{array}\right) \text { and } \sum_{t \in T} \frac{\lambda_{t}}{\sum_{t \in T} \lambda_{t}}=1
$$

It follows that

$$
\boldsymbol{0}_{n+1} \in \operatorname{conv}\left\{\left(\begin{array}{l}
\boldsymbol{a}_{t} \\
b_{t}
\end{array}\right): t \in T\right\} \subset \operatorname{clconv}\left\{\left(\begin{array}{l}
\boldsymbol{a}_{t} \\
b_{t}
\end{array}\right): t \in T\right\} .
$$

This means that $\pi$ does not satisfy the strong Slater condition, which is a contradiction. Thus $\sum_{t \in T} \lambda_{t}=0$. Since $\lambda$ is arbitrary, we conclude that $\Lambda^{*}=\{\lambda \equiv 0\}$.

Next, we present another proof of Theorem 4.3.

Proof. The first part of the already established proof shows that $\Lambda^{*}$ is bounded, $\Lambda^{*} \neq \emptyset$ and $v^{D}(\pi)=0$.

Now, suppose there exists $\lambda \in \Lambda^{*}$ such that $\sum_{t \in T} \lambda_{t}>0$. If $\delta>0$, we have $\delta \lambda \in \Lambda^{*}$. Hence, since $\lambda \in \Lambda^{*}$ and $c=\boldsymbol{0}_{n}$, we have

$$
\sum_{t \in T} \lambda_{t}\left(\begin{array}{c}
\boldsymbol{a}_{t} \\
b_{t}
\end{array}\right)=\left(\begin{array}{c}
\boldsymbol{0}_{n} \\
0
\end{array}\right)
$$

which implies that

$$
\sum_{t \in T} \delta \lambda_{t}\left(\begin{array}{c}
\boldsymbol{a}_{t} \\
b_{t}
\end{array}\right)=\delta \sum_{t \in T} \lambda_{t}\left(\begin{array}{c}
\boldsymbol{a}_{t} \\
b_{t}
\end{array}\right)=\delta\left(\begin{array}{c}
\boldsymbol{0}_{n} \\
0
\end{array}\right)=\left(\begin{array}{c}
\boldsymbol{0}_{n} \\
0
\end{array}\right) .
$$

On the other hand, $\|\delta \lambda\|_{1}=\delta\|\lambda\|_{1}>0$. If $\delta \rightarrow \infty$, then $\|\delta \lambda\|_{1} \rightarrow \infty$. This means that $\Lambda^{*}$ is not bounded, which contradicts Theorem 4.2.

In the case when $\boldsymbol{c} \neq \boldsymbol{0}_{n}$, we prove next that the infimum is strictly positive.

Theorem 4.4. Take $\pi=(\boldsymbol{a}, b, \boldsymbol{c})$ such that $\boldsymbol{c} \neq \boldsymbol{0}_{n}, \boldsymbol{c} \in M$ and $\pi$ satisfies the strong Slater condition. Then

$$
\inf \left\{\|\lambda\|_{1}: \lambda \in \Lambda^{*}\right\}>0 \text {. }
$$

Proof. Suppose that

$$
\inf \left\{\|\lambda\|_{1}: \lambda \in \Lambda^{*}\right\}=0 .
$$

Then there exists sequence $\left\{\lambda^{m}\right\}$ in $\Lambda^{*}$ such that

$$
\left\|\lambda^{m}\right\|_{1} \rightarrow 0
$$

i.e.,

$$
\sum_{t \in T} \lambda_{t}^{m} \rightarrow 0
$$


Since $\lambda^{m} \in \Lambda^{*}$, for all $m$, we have

$$
\sum_{t \in T} \lambda_{t}^{m}\left(\begin{array}{l}
\boldsymbol{a}_{t} \\
b_{t}
\end{array}\right)=\left(\begin{array}{c}
\boldsymbol{c} \\
{ }_{v} D(\boldsymbol{\pi})
\end{array}\right)
$$

Lemma 4.1 implies

$$
\sum_{i=1}^{n+2} \gamma_{t_{i}}^{m}\left(\begin{array}{l}
\boldsymbol{a}_{t_{i}} \\
b_{t_{i}}
\end{array}\right)=\left(\begin{array}{c}
\boldsymbol{c} \\
{ }^{D}(\pi)
\end{array}\right) \text { y } \sum_{i=1}^{n+2} \gamma_{t_{i}}^{m}=\sum_{t \in T} \lambda_{t}^{m} .
$$

Now, since $\sum_{t \in T} \lambda_{t}^{m} \rightarrow 0$ and $\left\{\left(\begin{array}{c}a_{t_{i}} \\ b_{t_{i}}\end{array}\right)\right\}$ are bounded sequences, taking $m \rightarrow \infty$ in (4.5) yields

$$
\sum_{i=1}^{n+2} 0\left(\begin{array}{c}
\boldsymbol{a}_{i} \\
b_{i}
\end{array}\right)=\left(\begin{array}{c}
\boldsymbol{c} \\
v^{D}(\boldsymbol{\pi})
\end{array}\right)
$$

(4.6) implies $\boldsymbol{c}=\boldsymbol{O}_{n}$, which is a contradiction.

Next we will exhibit several examples showing that the conditions that characterize the sets generated by the refined primal-dual partition in the continuous case, do not hold in the case of bounded coefficients.

We consider again the parameter of Example 4.1 and we show that the facts that $c \in$ int $M$ and that $\pi$ satisfes the strong Slater condition are not sufficient for ensuring that $\pi \in \Pi_{1}^{1}$.

Example 4.2. The primal problem is:

$$
\begin{array}{ll}
P_{2}: \quad & \operatorname{Min} \frac{1}{3} x_{1}+x_{2} \\
& x_{2} \geq 1 \\
\text { s.t. } \quad & t x_{1}+x_{2} \geq 0, \quad t \in(0,1), \\
& x_{1}+x_{2} \geq-1
\end{array}
$$

In [8], it was shown that $c^{2} \in$ int $M_{2}$ and that $\pi^{2}$ satisfies the Slater condition (in particular, $(0,2)^{\prime}$ is a Slater point). In fact, for $\varepsilon=\frac{1}{2},(0,2)^{\prime}$ is a strong Slater point. The latter would imply, in the continuous case, that $\pi^{2} \in \Pi_{1}^{1}$. However, in [8], it was shown that $v^{p}\left(\pi^{2}\right)=\frac{2}{3}$ and $F_{2}^{*}=\{(-1,1)\}$, but the dual problem

$$
\begin{gathered}
D_{2}: \quad \sup \lambda_{0}-\lambda_{1} \\
\text { s.t. } \quad \lambda_{0}\left(\begin{array}{l}
0 \\
1
\end{array}\right)+\sum_{t \in(0,1)} \lambda_{t}\left(\begin{array}{l}
t \\
1
\end{array}\right)+\lambda_{1}\left(\begin{array}{l}
1 \\
1
\end{array}\right)=\left(\begin{array}{l}
\frac{1}{3} \\
1
\end{array}\right) \\
\lambda \in \mathbb{R}_{+}^{(T)} .
\end{gathered}
$$

is not solvable. Therefore, $\pi^{2} \notin \Pi_{1}^{1}$ in the case of bounded coefficients.

The following example shows that the facts that $c \in$ int $M$ and that $\pi$ satisfies the strong Slater condition are not necessary conditions for $\pi$ to belong to $\Pi_{1}^{1}$.

Example 4.3. Take $\alpha>0$ and consider the following problem in $\mathbb{R}$ :

$$
\begin{array}{ll}
P_{3}: & \quad \inf \alpha x_{1} \\
& \text { s.t. } \quad t x_{1} \geq t^{2}, \quad t \in(0,1] .
\end{array}
$$


The problem is solvable, with

$$
F_{3}=\left\{x_{1}: x_{1} \geq 1\right\}, v^{P}\left(\pi^{3}\right)=\alpha \text { y } F_{3}^{*}=\left\{x_{1}=1\right\} .
$$

Also,

$$
c^{3}=\alpha \in \text { int } M_{3}=\text { int }(\text { cone }\{t: t \in(0,1]\})=\mathbb{R}_{+}^{0} .
$$

On the other hand, $(0,0)^{\prime} \in \operatorname{cl} \operatorname{conv}\left\{\left(t, t^{2}\right)^{\prime}: t \in(0,1]\right\}$, which implies that $\pi^{3}$ does not satisfy the strong Slater condition.

The dual problem is:

$$
\begin{gathered}
D_{3}: \quad \sup \sum_{t \in(0,1]} \lambda_{t} t^{2} \\
\text { s.t. } \sum_{\substack{t \in(0,1] \\
\lambda \in \mathbb{R}_{+}^{(T)} .}} \lambda_{t} t=\alpha \\
\quad \lambda=\alpha
\end{gathered}
$$

The function

$$
\lambda_{t}:= \begin{cases}\alpha, & \text { si } t=1 \\ 0, & \text { si }, 0<t<1\end{cases}
$$

is the only feasible solution and it is optimal. We conclude that $D_{3}$ is solvable with bounded optimal set. Hence, we have a parameter $\pi^{3} \in \Pi_{1}^{1}$, where $c^{3} \in$ int $M_{3}$, but $\pi^{3}$ does not satisfy the strong Slater condition.

In the particular case when $\boldsymbol{c}=\boldsymbol{0}_{n}$, we present a sufficient condition which implies that a given parameter $\pi=(\boldsymbol{a}, b, \boldsymbol{c})$ belongs to the set $\Pi_{1}^{1}$.

Corollary 4.2. Let $\pi=(\boldsymbol{a}, b, \boldsymbol{c})$ be a parameter with $\boldsymbol{c}=\boldsymbol{0}_{n}$ and $|T| \geq n+2$. If $\boldsymbol{c} \in$ int $M$ and $\pi$ satisfies the strong Slater condition, then $\pi \in \Pi_{1}^{1}$.

Proof. The result follows from Lemma 2.1 and Theorem 4.3.

As a consequence of Corollary 4.2, we have that the feasible set of the system $\left\{\boldsymbol{a}_{t}^{\prime} \boldsymbol{x} \geq b_{t}, t \in\right.$ $T\}$, (when $|T| \geq n+2$ ) is nonempty and bounded, if $\boldsymbol{0}_{n} \in$ int cone $\left\{\boldsymbol{a}_{t}, t \in T\right\}$ and there exist $\varepsilon>0$ and $\overline{\boldsymbol{x}} \in \mathbb{R}^{n}$ such that $\boldsymbol{a}_{t} \overline{\boldsymbol{x}}>b_{t}+\varepsilon$ for all $t \in T$. In fact, if these conditions hold, and we consider the parameter $\pi=\left(\boldsymbol{a}, b, \boldsymbol{0}_{n}\right)$, then $\pi \in \Pi_{1}^{1}$. In particular, $F^{*}$ is nonempty and bounded. Since in this case $F=F^{*}$, the result is immediate.

Corollary 4.3. Let $\pi=(\boldsymbol{a}, b, \boldsymbol{c})$ be a parameter with $b \equiv 0$ and $|T| \geq n+2$. If $\boldsymbol{c} \in$ int $M$ and $\pi$ satisfies the strong Slater condition, then $\pi \in \Pi_{1}^{1}$.

Proof. If $c \in M$ and $\pi$ satisfies the strong Slater condition, then, by Theorem $4.2, \Lambda^{*}$ is bounded. Now, since $c \in M$, we have that $\Lambda \neq \emptyset$. Furthermore, since $b \equiv 0$, every feasible solution of the dual problem is optimal. Hence, we get $\Lambda^{*} \neq \emptyset$. This implies that $\pi \in \Pi_{S}^{D}$. It only remains to prove that $\pi \in \Pi_{S}^{P}$, but this statement is equivalent to $c \in$ int $M$, if the parameter $\pi$ has consistent primal problem [4, Corollary 9.3.1]. Since $c \in$ int $M$, we only need to show that $\pi$ has a consistent primal problem, which holds true because, by hypothesis, $\pi$ satisfies the strong Slater condition. 
The parameter $\pi^{3}$ presented in Example 4.3 shows us that the condition presented in Theorem 4.1(ii), for the set $\Pi_{1}^{2}$, fails in the case of bounded coefficients. In particular, we show that the facts that $\left(\begin{array}{c}\boldsymbol{0}_{n} \\ 1\end{array}\right) \notin \operatorname{cl} N$, that $\boldsymbol{c} \in$ int $M$ and that $\pi$ does not satisfy the strong Slater condition, are not sufficient conditions for $\pi$ to belong to $\Pi_{1}^{2}$ In fact, we have that $\left(\begin{array}{c}\boldsymbol{o}_{n} \\ 1\end{array}\right) \notin c l N_{3}$ (because the primal problem is consistent), $c^{3} \in i n t M_{3}$ and $\pi^{3}$ does not satisfy the strong Slater condition, but $\pi^{3} \notin \Pi_{1}^{2}$.

The parameter $\pi^{2}$ of Example 4.2 also shows that the facts that $\left(\begin{array}{c}\boldsymbol{0}_{n} \\ 1\end{array}\right) \notin c l N$, that $c \in$ int $M$ and that $\pi$ does not satisfy the strong Slater condition are not necessary conditions for $\pi$ to belong to $\Pi_{1}^{2}$. Indeed, $\pi^{2} \in \Pi_{1}^{2},\left(\begin{array}{c}\boldsymbol{o}_{n} \\ 1\end{array}\right) \notin c l N_{2}$ and $c^{2} \in$ int $M_{2}$, but $\pi^{2}$ satisfies the Slater condition.

With the next example we show that the condition presented in Theorem 4.1 (iii) is not valid in the case of bounded coefficients. In particular, it shows that the facts that $c \in M \backslash$ int $M$ and that $\pi$ satisfies the strong Slater condition are not necessary conditions for $\pi$ to belong to $\Pi_{1}^{3}$.

Example 4.4. Consider the problem in $\mathbb{R}^{2}$ defined by:

$$
\begin{array}{ll}
P_{4}: & \quad \inf x_{1}+x_{2} \\
& \text { s.t. } \quad t^{2} x_{1}+t x_{2} \geq t, \quad t \in(0,1] .
\end{array}
$$

The feasible set is shown in Figure 1.

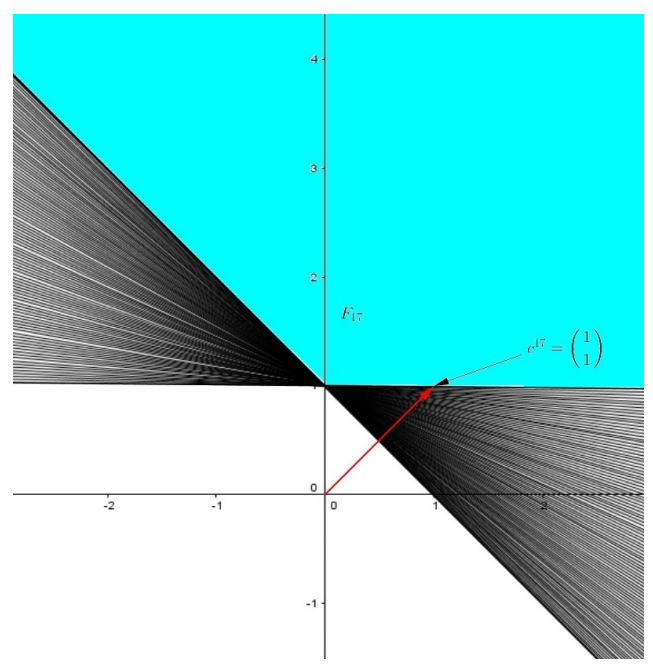

FIGURE 1. Feasible set of $P_{4}$

In view of Figure 1, we have,

$$
v^{P}\left(\pi^{4}\right)=1
$$

and

$$
F_{4}^{*}=\left\{\left(\begin{array}{l}
x_{1} \\
x_{2}
\end{array}\right): x_{2}=1-x_{1} \text { and } x_{1} \leq 0\right\} .
$$

The problem is solvable with unbounded optimal set. On the other hand, it holds that

$$
\left(\begin{array}{l}
0 \\
0 \\
0
\end{array}\right) \in \operatorname{clconv}\left\{\left(\begin{array}{l}
t^{2} \\
t \\
t
\end{array}\right): t \in(0,1]\right\},
$$


which implies that $\pi^{4}$ does not satisfy the strong Slater condition. Figure 2 shows that

$$
c^{4}=\left(\begin{array}{l}
1 \\
1
\end{array}\right) \in M_{4} \backslash \text { int } M_{4}
$$

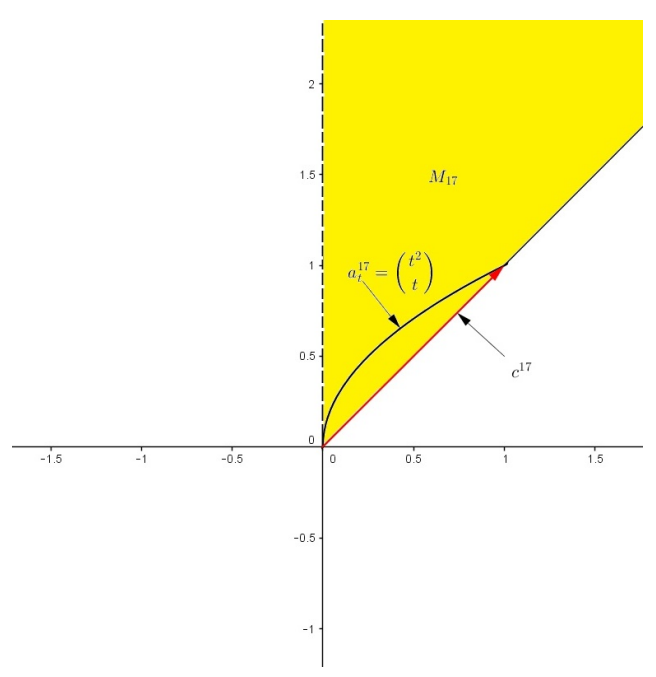

Figure 2. Cone $M_{4}$ of $P_{4}$

The dual problem is:

$$
\begin{array}{cc}
D_{4}: \quad & \sup \sum_{t \in(0,1]} \lambda_{t} t \\
\text { s.t. } & \sum_{t \in(0,1]} \lambda_{t}\left(\begin{array}{c}
t^{2} \\
t
\end{array}\right)=\left(\begin{array}{l}
1 \\
1
\end{array}\right) \\
& \lambda \in \mathbb{R}_{+}^{(T)} .
\end{array}
$$

The function

$$
\lambda= \begin{cases}1, & \text { si } t=1, \\ 0, & \text { si, } 0<t<1,\end{cases}
$$

is the only feasible solution and it is also optimal. It implies that $D_{4}$ is solvable and it has a bounded optimal set. Hence, $\pi^{4} \in \Pi_{1}^{3}$ and although

$$
c^{4} \in M_{4} \backslash \text { int } M_{4},
$$

we have that $\pi^{4}$ does not satisfy the strong Slater condition.

In the following example, we show that the facts that $c \in M \backslash$ int $M$ and that $\pi$ satisfies the strong Slater condition are not sufficient for $\pi$ to belong to $\Pi_{1}^{3}$.

Example 4.5. Consider the following problem in $\mathbb{R}^{2}$ :

$$
\begin{array}{ll}
P_{5}: \quad & \text { inf } x_{2} \\
& \\
\text { s.t. } & x_{2} \geq t, \quad t \in[0,1), \\
& x_{1} \geq 0 .
\end{array}
$$




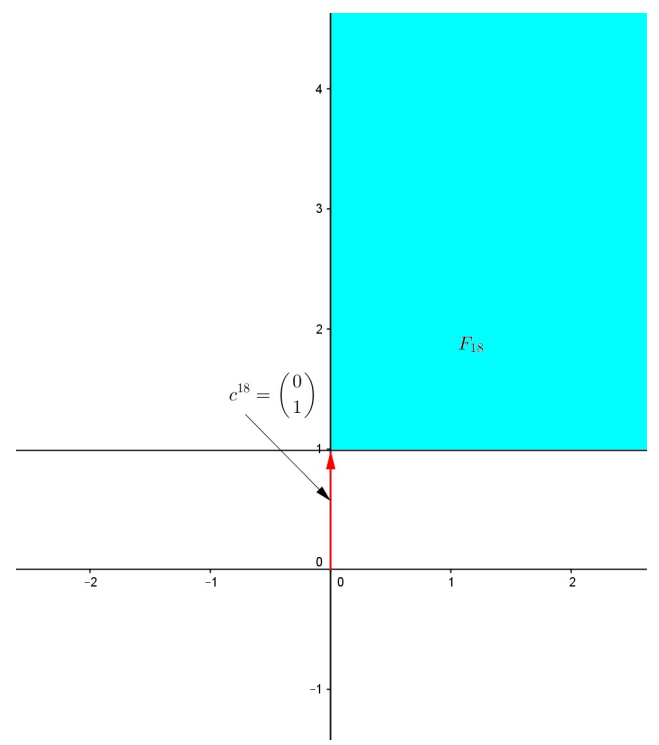

FIGURE 3. Feasible set of $P_{5}$

The feasible set is shown in Figure 3. We have that

$$
v^{P}\left(\pi^{5}\right)=1
$$

and

$$
F_{5}^{*}=\left\{\left(\begin{array}{l}
x_{1} \\
x_{2}
\end{array}\right): x_{2}=1, x_{1} \geq 0\right\} .
$$

The problem is solvable with unbounded optimal set. On the other hand, $\pi^{5}$ satisfies the strong Slater condition. Indeed, take $\varepsilon=1$ and consider $\bar{x}=\left(\begin{array}{l}3 \\ 3\end{array}\right)$. Now, Figure 4 shows that $c^{5}=$ $(0,1)^{\prime} \in M_{5} \backslash$ int $M_{5}$.

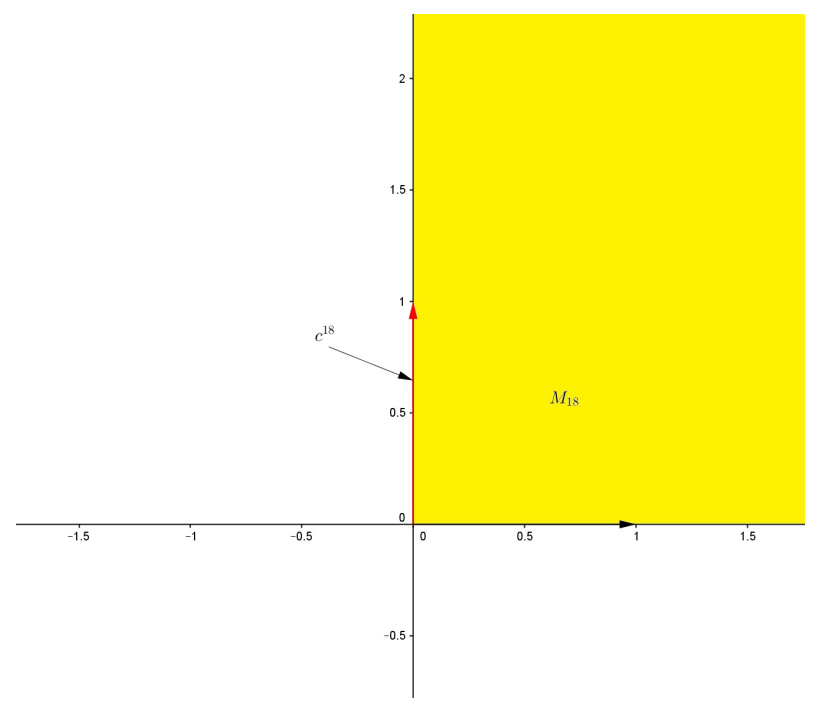

FIGURE 4. Cone $M_{5}$ of $P_{5}$ 
The dual problem is:

$$
\begin{array}{cl}
D_{5}: & \sup \sum_{t \in[0,1)} \lambda_{t} t \\
\text { s.t. } & \sum_{t \in[0,1)} \lambda_{t}\left(\begin{array}{l}
0 \\
1
\end{array}\right)+\lambda_{1}\left(\begin{array}{l}
1 \\
0
\end{array}\right)=\left(\begin{array}{l}
0 \\
1
\end{array}\right) \\
\lambda \in \mathbb{R}_{+}^{(T)} .
\end{array}
$$

In view of the constraints, we get that

$$
\sum_{t \in[0,1)} \lambda_{t}=1
$$

and

$$
\lambda_{1}=0
$$

Let us consider the sequence of feasible points $\bar{\theta}^{m}=\left(\lambda^{m} ; \lambda_{1}\right)$, where $\lambda_{1}=0$ and $\lambda^{m}$ is defined by

$$
\lambda_{t}^{m}:= \begin{cases}1, & \text { if } t=1-\frac{1}{m}, \\ 0, & \text { if, } t \in[0,1) \backslash \frac{1}{m}\end{cases}
$$

It follows that

$$
\sum_{t \in[0,1)} \lambda_{t}^{m} t=1-\frac{1}{m}
$$

approaches 1 as $m$ tends to infinity. This implies that $v^{D}\left(\pi^{5}\right)=1$, but $\Lambda^{*}=\emptyset$, so that the dual problem is not solvable. So, we have the parameter $\pi^{5}$ for which $c^{5} \in M_{5} \backslash$ int $M_{5}$ and $\pi^{5}$ satisfies the strong Slater condition, but $\pi^{5} \notin \Pi_{1}^{3}$.

In Example 4.5,

$$
\pi^{5} \in \Pi_{1}^{4}, \quad\left(\begin{array}{c}
\boldsymbol{0}_{n} \\
1
\end{array}\right) \notin c l N_{5} \text { y } c^{5} \in M_{5} \backslash \text { int } M_{5},
$$

but $\pi^{5}$ does not satisfy the strong Slater condition, which implies that the condition presented in Theorem 4.1(iv), for the set $\Pi_{1}^{4}$ does not remain valid in the case of bounded coefficients. In particular, this example shows that the facts that $\left(\begin{array}{c}\boldsymbol{0}_{n} \\ 1\end{array}\right) \notin c l N$, that $c \in M \backslash$ int $M$ and that $\pi$ does not satisfy the strong Slater condition, are not necessary conditions for $\pi$ to belong to $\Pi_{1}^{4}$.

In Example 4.4, $\left(\begin{array}{c}\boldsymbol{o}_{n} \\ 1\end{array}\right) \notin \mathrm{cl} N_{4}, \boldsymbol{c}^{4} \in M_{4} \backslash$ int $M_{4}$ and $\pi^{4}$ does not satisfy the strong Slater condition, but $\pi^{4} \notin \Pi_{1}^{4}$. Thus, we have shown that the facts that $\left(\begin{array}{c}\boldsymbol{o}_{n} \\ 1\end{array}\right) \notin \mathrm{cl} N$, that $c \in M \backslash$ int $M$ and that $\pi$ does not staisfy the strong Slater condition are not sufficient for $\pi$ to belong to $\Pi_{1}^{4}$.

Following similar arguments to those in [5], we conclude that $\Pi_{5}^{1}=\emptyset$ in the case of bounded coefficients. We have thus that $\Pi_{5}=\Pi_{N}^{P} \cap \Pi_{I C}^{D}$.

In the following example, we show that $\Pi_{6}^{1}=\Pi_{I C}^{P} \cap \Pi_{S}^{D} \neq \emptyset$ in the case of bounded coefficients, which is not possible in the continuous case.

Example 4.6. Consider the problem in $\mathbb{R}^{2}$ given by:

$$
\begin{aligned}
& P_{6}: \quad \text { inf } 0 \\
& \text { s.t. } t x_{1}+t x_{2} \geq 1, \quad t \in(0,1] .
\end{aligned}
$$


The problem is inconsistent because

$$
\left(\begin{array}{l}
0 \\
0 \\
1
\end{array}\right)=c l \text { cone }\left\{\left(\begin{array}{l}
t \\
t \\
1
\end{array}\right), t \in(0,1]\right\} .
$$

The dual problem is:

$$
\begin{gathered}
D_{6}: \quad \sup _{t \in(0,1]} \lambda_{t} \\
\text { s.t. } \sum_{\substack{t \in(0,1] \\
\lambda \in \mathbb{R}_{+}^{(T)}}} \lambda_{t}\left(\begin{array}{l}
t \\
t
\end{array}\right)=\left(\begin{array}{l}
0 \\
0
\end{array}\right) \\
\lambda \in{ }^{(T)}
\end{gathered}
$$

In view of the constraints, we have

$$
\sum_{t \in(0,1]} \lambda_{t} t=0
$$

Since $t \in(0,1]$, the equality in (4.7) is only possible if

$$
\sum_{t \in(0,1]} \lambda_{t}=0
$$

It follows that

$$
v^{D}\left(\pi^{6}\right)=0 \text { y } \Lambda_{6}^{*}=\{\lambda \equiv \overline{0}\} .
$$

Therefore, $D_{6}$ is bounded and solvable with bounded optimal set.

The set $\Pi_{6}^{2}$ is also nonempty, even in the continuous case.

We close this section ends with the presentation of a few necessary conditions, which follow from already known results. The following two result follow from [4, Corollary 9.3.1] and Corollary 4.1.

- If $\pi \in \Pi_{1}^{2}$ and $\Lambda^{*}$ is unbounded, then $c \in$ int $M$ and $\pi$ does not satisfy the strong Slater condition.

- If $\pi \in \Pi_{1}^{4}$ and $\Lambda^{*}$ is unbounded, then $c \in M \backslash i n t M$ and $\pi$ does not satisfy the strong Slater condition.

The next result follows from [7].

- If $\pi \in \Pi_{1}^{2}$ and $\Lambda^{*}=\emptyset$, then there exists a sequence $\left\{\pi^{r}\right\}$ in $\Pi$ such that $\pi^{r} \rightarrow \pi, c^{r} \in$ int $M_{r}$ and $\pi^{r}$ satisfies the strong Slater condition.

We conclude by mentioning that we have obtained a sufficient condition for the boundedness of the dual optimal set, which might however be empty. Conditions that guarantee the solvability of the dual problem turn out to be hard to establish, even in the continuous case. This is left as a challenging problem for future research.

\section{Acknowledgements}

This research of the first author was supported by CONACYT, Mexico. This research of the second author was supported by Sistema Nacional de Investigadores, Mexico. This research of the forth author was partially supported by MINECO of Spain and ERDF of EU, Grant MTM2014-59179-C2-1-P, and Sistema Nacional de Investigadores, Mexico. 


\section{REFERENCES}

[1] A.B. Barragán, L.A. Hernández, M.I. Todorov, New primal-dual partition of the space of linear semi-infinite continuous optimization problems, Comptes Rendus de l'Academie Bulgare des Sciences, 69 (2016), 1263 1274.

[2] M. Bazaraa, Programación lineal y flujo en redes, Limusa, México, 2005.

[3] M.A. Goberna, V. Jornet, R. Puente, Optimización Lineal. Teoría, Métodos y Modelos, Mc Graw Hill, España, 2004.

[4] M.A. Goberna, M, López, Linear Semi-infinite Optimization, John Wiley and Sons, England, 1998.

[5] M.A. Goberna, M.I. Todorov, Generic Primal-dual solvability in continuous linear semi-infinite optimization, Optimization, 57 (2008), 239-248.

[6] M.A. Goberna, M.I. Todorov, Primal-dual stability in continuous linear optimization, Math. Program. 116B (2009), 129-147.

[7] L.A. Hernández, Representaciones del conjunto factible y estabilidad del problema dual en programación lineal semi-infinita, Tesis de doctorado, FCFM-BUAP, Puebla, México, 129-147, 2004.

[8] P.D. Ochoa, V.N. Vera de Serio, Stability of the primal-dual partition in linear semi-infinite programming, Optimization, 61 (2012), 1449-1465.

[9] R.T. Rockafellar, Convex Analysis, Princeton University Press, Princeton, 1970. 